\title{
Light-induced Polarization-directed Growth of Optically Printed Gold Nanoparticles
}

Ianina L. Violi ${ }^{1}$, Julián Gargiulo ${ }^{1}$, Catalina von Bilderling ${ }^{1}$, Emiliano Corté ${ }^{1 \dagger}$, Fernando D. Stefani $* 1,2$

${ }^{1}$ Centro de Investigaciones en Bionanociencias (CIBION), Consejo Nacional de Investigaciones Científicas y Técnicas (CONICET), Godoy Cruz 2390, C1425FQD Ciudad de Buenos Aires, Argentina

${ }^{2}$ Departamento de Física, Facultad de Ciencias Exactas y Naturales, Universidad de Buenos Aires, Güiraldes 2620, C1428EAH Ciudad de Buenos Aires, Argentina

\section{ABSTRACT}

Optical printing has been proved a versatile and simple method to fabricate arbitrary arrays of colloidal nanoparticles (NPs) on substrates. Here we show that is also a powerful tool for studying chemical reactions at the single NP level. We demonstrate that $60 \mathrm{~nm}$ gold NPs immobilized by optical printing can be used as seeds to obtain larger NPs by plasmon-assisted reduction of aqueous $\mathrm{HAuCl}_{4}$. The final size of each $\mathrm{NP}$ is simply controlled by the irradiation time. Moreover, we show conditions for which the growth occurs preferentially in the direction of 
light polarization, enabling the in-situ anisotropic reshaping of the NPs in pre-determined orientations.

KEYWORDS Nanofabrication, optical forces, plasmon assisted chemistry, polarization

TEXT

Nowadays, a vast catalogue of nanomaterials can be fabricated through colloidal synthesis ${ }^{1}$, where metallic NPs have a remarkable position because of their unique optical and electronic properties ${ }^{2}$. Those properties are tightly related to their size, composition and morphology $y^{3-5}$. Typical NP synthesis procedures are highly sensitive to small variations in the reaction conditions. This is on one hand advantageous, because it enables the variation of NP size and morphology by performing small changes in the reaction settings. On the other hand, establishing robust procedures for colloidal synthesis of NPs generally requires laborious and systematic efforts ${ }^{6,7}$. Typically, directing the outcome of a colloidal synthesis requires precise control over concentration of reagents, as well as other factors such as agitation and temperature. For instance, tight control over the well-known Turkevich ${ }^{8}$ reaction introduced more than fifty years ago remained elusive until very recently ${ }^{9,10}$. Moreover, in the case of metallic nanoparticles, the presence of light can dramatically change the outcome of a synthesis. It has been found that NP reshaping and growth can be induced and controlled by light, in general tuned to the surface plasmon resonances of the NPs ${ }^{11-18}$. These reactions are a special case of plasmon-assisted chemistry, where the photophysical processes related to the excitation of localized surface plasmon resonances drive chemical reactions on the nanoscale ${ }^{17-20}$. These processes can be categorized as 
i) local heating, ii) near-field enhancement, and iii) plasmon-decay generated hot-electrons and hot-holes that can react with nearby molecular species.

Optical printing is an emerging and versatile method for the selective deposition of colloidal NPs on specific locations of a substrate using optical forces ${ }^{21-24}$. It has great potential as a tool to incorporate the vast variety of colloidal NPs into NP-based devices and circuits. This has motivated the investigation of the nanoscale interactions involved during the process of optical printing so as to control the precision ${ }^{21}$, orientation ${ }^{22}$ and type $\mathrm{e}^{23}$ of the printed NP, as well as the inter-particle distance ${ }^{25}$.

The combination of optical printing and plasmon assisted chemistry is appealing for two main reasons. First, it facilitates the systematic investigations of chemical reactions on immobilized single NPs, where the effect of a controlled dosage of light, including the elusive role of light polarization ${ }^{18}$ can be studied. Second, it would enable the fabrication of NPs-based devices, where the properties of each component could be fine-tuned by plasmon assisted growth. Here, we show the first example of plasmon-assisted and polarization-directed growth of Au NPs from spheres to spheroids, using optical printing of colloidal NPs as the starting point to tailor NP size and shape. Individual Au NPs on an array were grown in a controlled fashion to different final sizes by illumination at their surface plasmon resonance in presence of a $\mathrm{HAuCl}_{4}$ aqueous solution. As they grow, the NPs reshape their morphology from nearly spherical to spheroidal with their major axis parallel to the polarization of the light used to induce the reaction.

The essential components of the experimental setup are depicted in Figure 1A. Briefly, a custom-made dark-field optical microscope was modified to incorporate a $\mathrm{CW}$ laser at $532 \mathrm{~nm}$, focused on the substrate to its diffraction limit with a water-immersion 1.0 NA objective. A 
confocal detection channel for elastic scattering was implemented using a notch filter and an amplified photodiode. Nanometric sample scanning and positioning was achieved with a XYZ piezo-electric stage. For dark-field illumination we used a halogen lamp and a dark-field condenser. Images were acquired using a digital color photo camera. Single NP scattering spectra were obtained with a spectrometer based on 300 lines/mm diffraction grating and a CCD device. A polarizer in a rotation mount allowed selecting the polarization direction of the scattered light at the entrance of the spectrometer. Further technical details are provided in the SI.

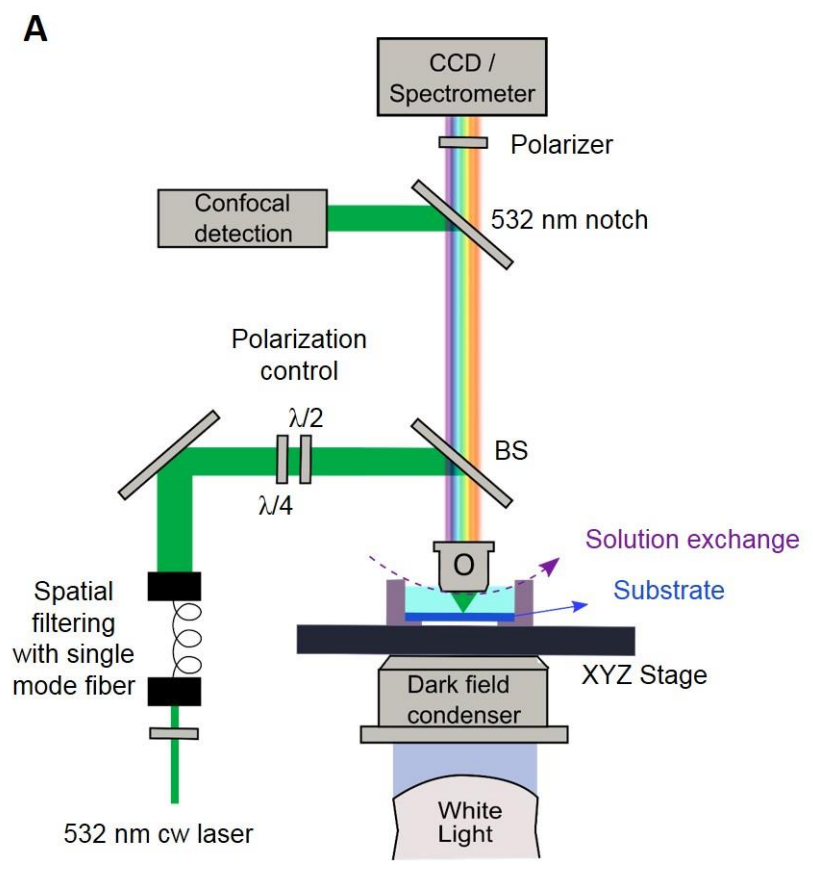

B

1) Optical printing single AuNPs from colloidal suspension

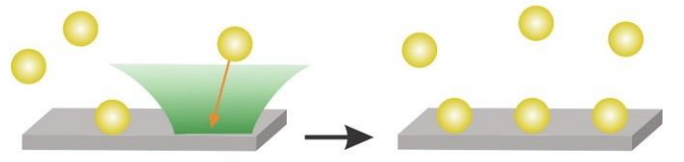

2) Exchange of AuNP colloid for $\mathrm{HAuCl}_{4}$ (aq)

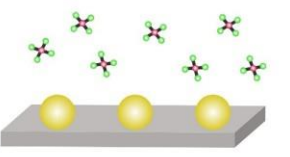

3) Light-induced growth of AuNPs one by one

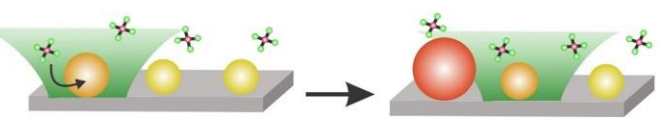

Figure 1. A) Schematic of the experimental setup. B) Essential steps of the light-induced growth experiment: 1) $60 \mathrm{~nm} \mathrm{Au} \mathrm{NPs} \mathrm{are} \mathrm{optically} \mathrm{printed} \mathrm{from} \mathrm{colloidal} \mathrm{suspension;} \mathrm{2)} \mathrm{the} \mathrm{Au} \mathrm{NPs}$ suspension is removed, the sample chamber is rinsed thoroughly with ultrapure water, and filled with an aqueous solution of $\mathrm{HAuCl}_{4}$; 3) Each Au NP is irradiated individually with light of 532 nm for a given time interval. 
Each experiment of light-induced NP growth involved essentially three steps (Fig. 1B). In the first step, a regular grid of Au NPs of $60 \mathrm{~nm}$ in diameter was fabricated on a glass substrate by optical printing single Au NPs from colloidal suspension. Details about the optical printing procedure can be found in our previous report ${ }^{25}$ and in the SI. The second step was the exchange of the $\mathrm{Au} \mathrm{NP}$ colloid by an aqueous solution of $\mathrm{HAuCl}_{4}$, including a thorough rinsing with ultrapure water in between. Finally, each NP of the grid was localized by a confocal scan, moved to the center of the laser focus, and irradiated with linearly polarized light of $532 \mathrm{~nm}$ during a given time period. The first and third steps were fully automated by PC control.

Figure 2 shows dark-field micrographs of a typical grid of Au NPs before (Fig. 2A) and after (Fig 2B) a light-induced growth experiment using $0.9 \mathrm{MW} / \mathrm{cm}^{2}$ of $532 \mathrm{~nm}$ laser light and $1.5 \mu \mathrm{M} \mathrm{HAuCl} 4$. The Au NPs on each column were irradiated for a different time interval. The controlled light induced growth is evident already from a visual inspection of the dark-field images. As the irradiation time increases, the scattering by the NPs becomes more intense and turns from green to orange. We monitored the printed Au NPs for several days in the absence either of light or gold precursor, and no changes whatsoever were observed (see Figure S1).

Further insight is obtained by analyzing the scattering spectra of the individual NPs. Light scattered by the NPs was analyzed using a polarizer filter in order to separate the contributions polarized parallel (PX) and perpendicular (PY) to the electric field vector used for the growth reaction. Example spectra of a control NP, and a NP after $210 \mathrm{~s}$ of irradiation are shown in Figures $2 \mathrm{C}$ and 2D, respectively. The scattering spectra of Au NPs before irradiation are typical of near spherical Au NPs; they present a single peak at around $555 \mathrm{~nm}$, independent of the polarization (Figure 2C). Remarkably, the scattering spectra of light-grown Au NPs present different contributions depending on polarization. The scattering maximum of the PX contribution is 
considerably red-shifted, peaking at $620 \mathrm{~nm}$. The PY spectrum, with its maximum at $570 \mathrm{~nm}$ remains closer to the original scattering peak (Figures 2D). Both PX and PY scattering spectra of the grown Au NPs are broader in comparison to the spectra of Au NPs before irradiation. This constitutes a strong indication that the light induced growth occurred preferentially in the direction determined by the electric field of the light used for the growth reaction. In Figure 2E we show the average evolution of the PX and PY scattering peak wavelengths as a function of the irradiation time. Each data point corresponds to the average obtained from $11 \mathrm{Au}$ NPs. At first, and up to approximately $30 \mathrm{~s}$, the scattering peak wavelengths of both polarizations increase together, indicating that the growth is rather isotropic during the first stages of the reaction. Then, at around $60 \mathrm{~s}$, the PY peak wavelength reaches a plateau and does not increase with further irradiation, while the PX peak continuously shifts to longer wavelengths. 
A Before irradiation
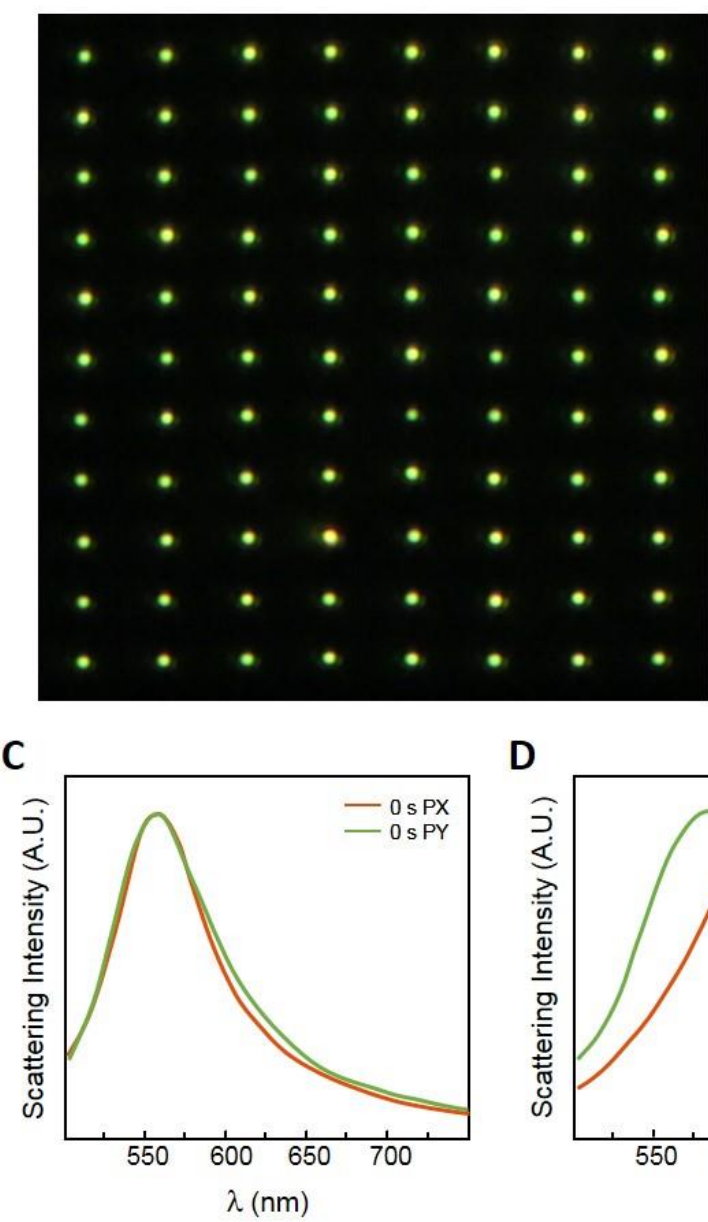

B Irradiation time (s)
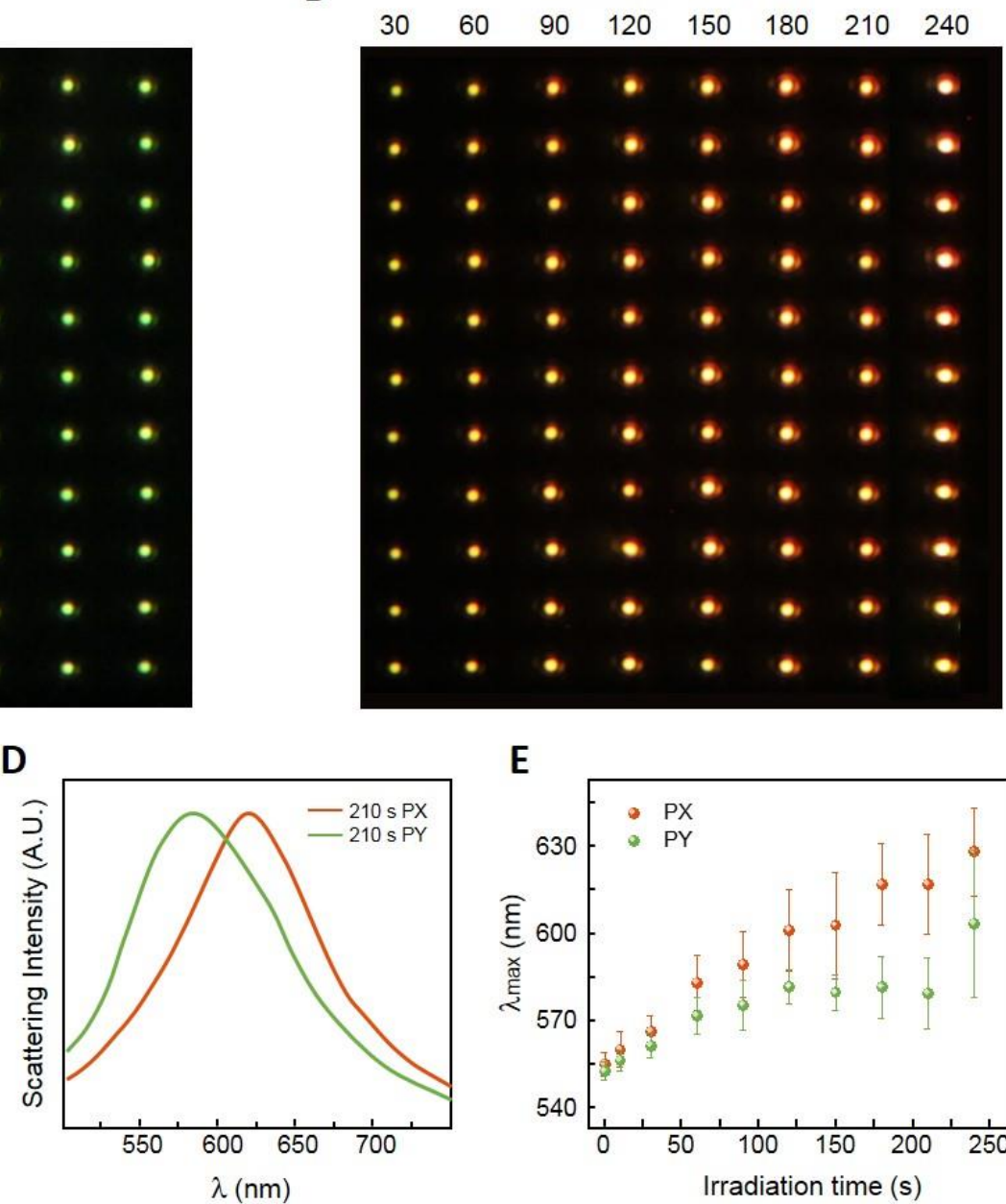

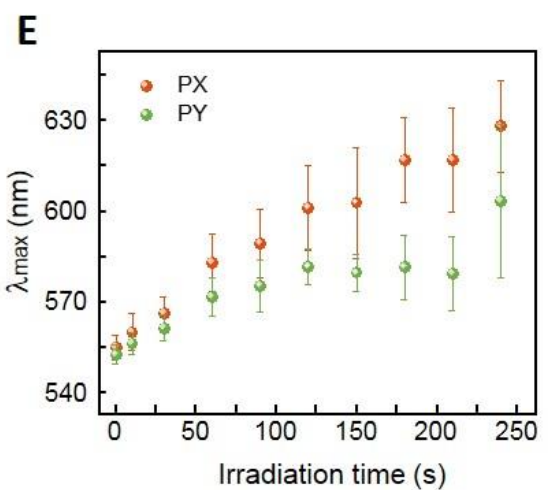

Figure 2. A) Dark field image of the optically printed Au NPs grid. B) Dark field image of the same grid of Au NPs after each column was irradiated with $532 \mathrm{~nm}$ light for different times in the presence of $1.5 \mu \mathrm{M} \mathrm{HAuCl}_{4}(\mathrm{aq})$. C) Typical scattering spectra of a single Au NP before the light induced growth, measured with a polarization filter aligned parallel (PX) and perpendicular (PY) to the electric field used for growth. D) Typical PX and PY scattering spectra of a single Au NP irradiated for 210 s. E) Peak wavelength $(\lambda \max )$ of the PX and PY scattering spectra as a function of the irradiation time. Each data point corresponds to the average of $11 \mathrm{Au} \mathrm{NP}$; error bars indicate the standard deviation. 
The morphology of the Au NPs was then characterized individually by field emission scanning electron microscopy (FE-SEM). Representative images of the Au NPs grown for different irradiation times are presented in Figure 3A. The FE-SEM observations confirm the predictions based on the scattering spectra. Upon irradiation, the Au NPs change from nearly spherical to spheroidal with their major diameter parallel to the polarization of the light used for the growth reaction. In order to quantify the evolution of the reshaping, we analyzed the FE-SEM images of all single Au NPs, identified their minor and major diameters and determined the angle between them ( $\alpha$, Figure 3). Figure 3B shows the average minor and major diameters of the $\mathrm{Au}$ NPs as a function of the irradiation time, and Figure 3C the average angle $\alpha$. The Au NPs increase their size, first in a rather isotropic way, and then faster in the direction imposed by the electric field. The directionality imposed by the polarization of light is evident as well in the reduced dispersion of $\alpha$ as the light-induced growth reaction progresses. At short irradiation times, values of $\alpha$ spanning a range of almost $90^{\circ}$ are observed, reflecting the fact that the Au NPs are nearly spherical without any preferential orientation. As the growth reaction advances, $\alpha$ takes values over a much narrower range of less than $\pm 10^{\circ}$, indicating the Au NPs have grown preferentially in the direction imposed by polarization.

The mechanistic details of light-induced growth of metallic NPs are not trivial and are still a matter of investigation and debate, even after more than a decade since its discovery with silver $\mathrm{NPs}^{18,26}$. Light-induced growth of gold NPs has only recently been reported in a detailed study, using various reducing agents and polyvinylpyrrolidone (PVP) as surface capping ${ }^{27}$. Currently, the proposed mechanism ${ }^{28}$ involves the following steps. Upon illumination, hot electron-hole pairs are generated in the metallic NP, preferentially in regions of enhanced electric field (hot-spots) determined by the particular plasmon mode excited. Such hot electron-hole pairs have a lifetime 
of the order of femtoseconds ${ }^{29}$, and therefore are too short lived to directly drive the reduction reaction of metal ions in solution. Instead, it is proposed that molecules at the NP surface can act as electron donors to fill the photo-generated holes, rendering the NPs with a net negative charge, which has been measured consistently ${ }^{16,26}$. Then, metallic ions from the solution that get in contact with the NP are reduced and incorporated one by one to the NP, leading to growth with crystalline order. A reductive agent present in the solution, usually citrate, closes the redox cycle. The final NP shape is independent of the positions of the plasmonic hotspots obtained by varying the incident-light polarization ${ }^{16}$ or wavelength ${ }^{27}$, which is in contrast to our findings.

Our experiments however are conducted under different conditions from most previous reports. Although the Au NPs had a surface layer of citrate anions, no extra citrate or any other reducing agent was present in the solution; i.e. the redox cycle is probably closed by water oxidation $^{30,31}$. Experiments conducted in the presence of citrate led to very rapid and uncontrolled growth of the printed NPs, as well as to the formation of small Au nanoparticles that uniformly deposit on the substrate even in the absence of light (data not shown). In addition, we used irradiances that are $10^{9}$ times higher than what has been typically used. These conditions in turn modify several critical experimental parameters: i) much more intense near fields are generated, ii) the Au NP surface and the solution around are at temperatures considerably higher than room temperature during the growth reaction $\left(\mathrm{ca} .210^{\circ} \mathrm{C}\right.$, see $\mathrm{SI}$ ), iii) reaction rates are higher in comparison to previous reports ( 4 min vs. hours respectively), even when the reducing agent is weaker. There are few examples of experiments conducted in comparable conditions ${ }^{32,33}$. For example, Kim et. al. ${ }^{31}$ recently described the reduction of aqueous platinum ions onto Au NPs observing a preferential deposition in the NPs hot spots, in agreement with our findings. 
A

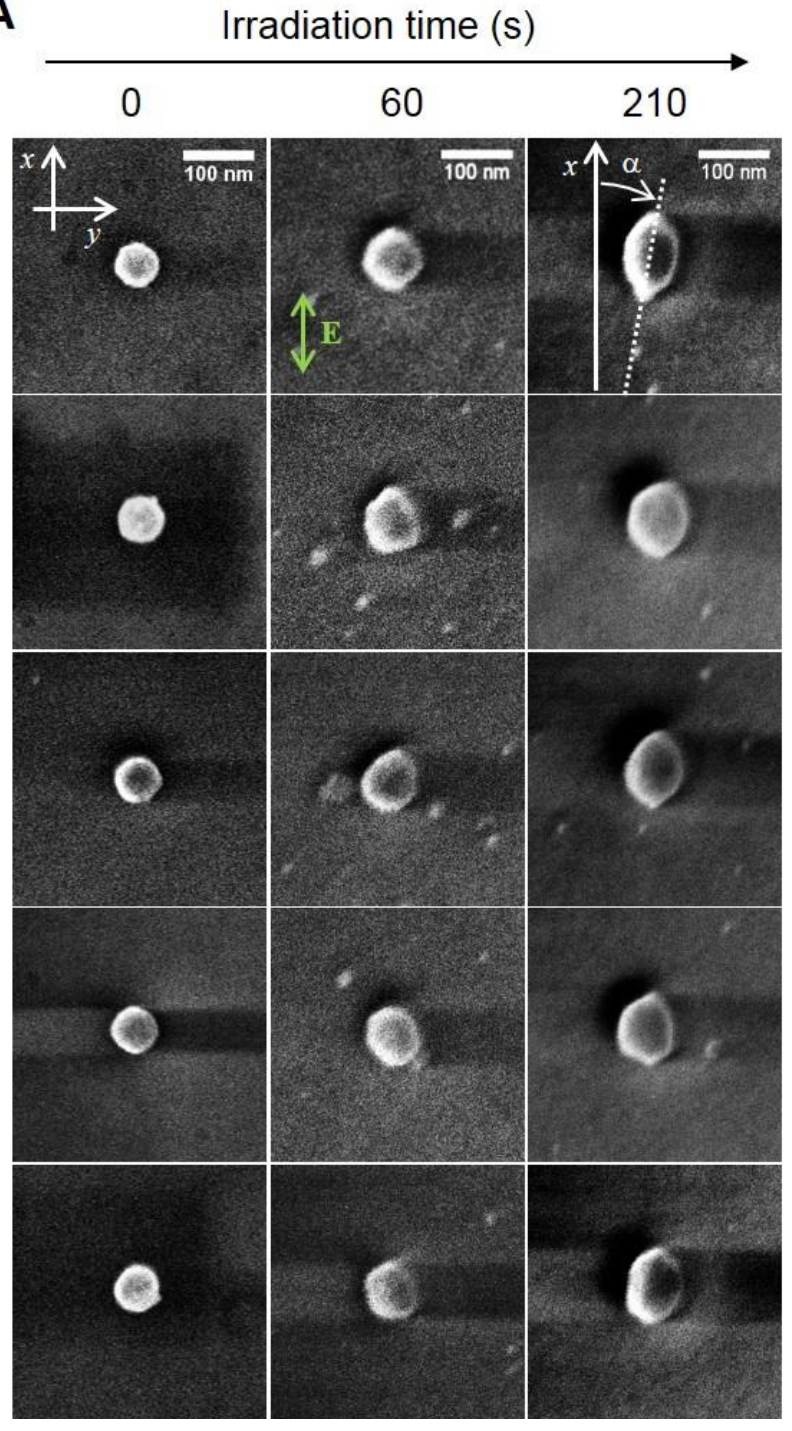

B

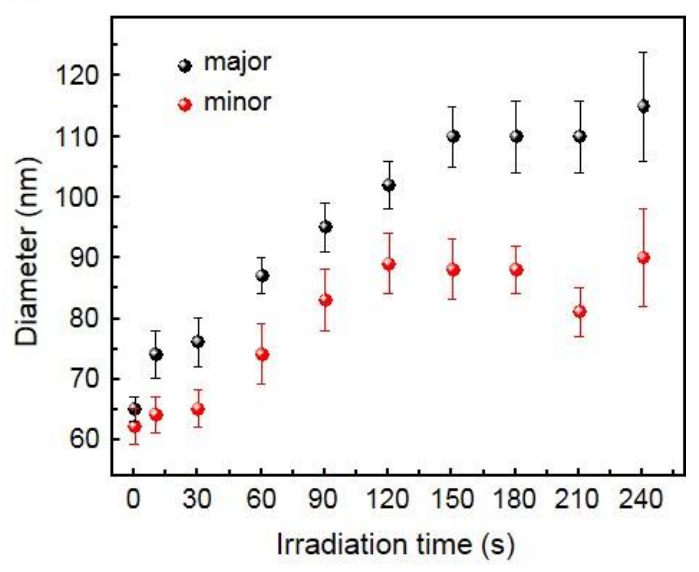

C

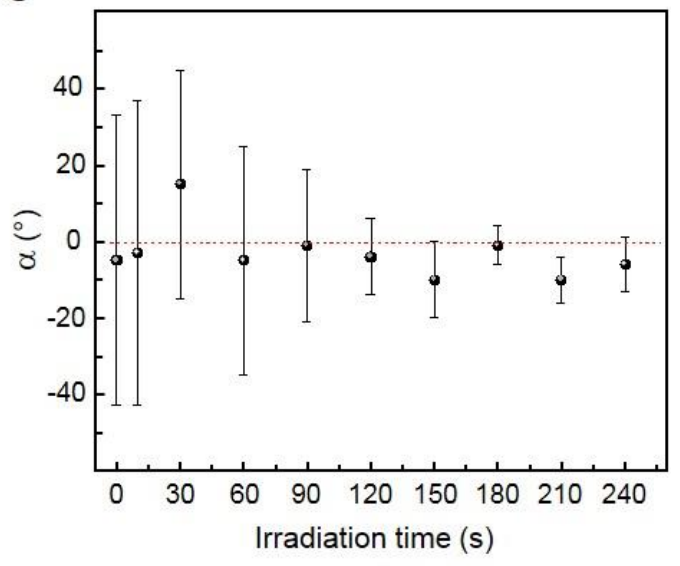

Figure 3. A) FE-SEM images of five representative $\mathrm{Au}$ NPs in the presence of $\mathrm{HAuCl}_{4}$ without irradiation ( $0 \mathrm{~s}$ ), and irradiated for $60 \mathrm{~s}$ and $210 \mathrm{~s}$ using a $532 \mathrm{~nm}$ focused laser. Electric field polarization vector $(E)$, angle description and axis references are also depicted. B) Major and minor diameter dependence on the irradiation time using linearly polarized light along X. C) Angle obtained between $\mathrm{X}$ and the major axis of the NPs $(\alpha)$ vs. irradiation time. 
From the FE-SEM images, it is evident that the Au NPs do not grow following any crystallographic order, which is consistent with the fast growing rates ${ }^{34}$ and the absence of surface molecules with preferential selectivity to crystallographic planes (such as PVP). The anisotropic growth must have a different origin. In order to gain insight into the process we performed some additional experiments. We noted in some cases, that smaller Au NPs $(5-15 \mathrm{~nm})$ appeared on the irradiated area near the printed Au NPs (e.g. Figure 3A $60 \mathrm{~s}$ and $210 \mathrm{~s}$ ). For the longest exposure time, the amount of these satellite NPs was considerable. Scattering by these NPs is most probably the reason why the points at $240 \mathrm{~s}$ in Figure 2E deviate from the trend. Based on these observations, we irradiated regions of the substrate far from any Au NP. Using the same irradiance as for the NP growth produced no effect whatsoever. However, using irradiances 9- to 18 -fold higher (8 to 16 $\mathrm{MW} / \mathrm{cm}^{2}$ ), smaller Au NPs deposited and grew on any region of the substrate (see Figure S2). Noting that both the substrate and the $\mathrm{HAuCl}_{4}$ solution are practically transparent at $532 \mathrm{~nm}$, we speculate that small Au clusters form and disassemble in a dynamic equilibrium. Au clusters of a few tens of atoms present broad visible absorption ${ }^{35}$. NPs as small as $2.9 \mathrm{~nm}$ in diameter $(\sim 700$ atoms) already sustain a surface plasmon resonance and absorb resonantly light of $532 \mathrm{~nm}^{35}$. Such spontaneously formed clusters are the ones interacting with light and driving the reaction. The high levels of irradiance needed for these clusters to drive the reduction of $\mathrm{HAuCl}_{4}$ are comparable to what is expected near the poles of the immobilized $60 \mathrm{~nm}$ (and larger) Au NPs when they are plasmonically excited with polarized light (Figure S2 C). Therefore, we interpret that the anisotropic NP growth induced by the polarization of light occurs via small Au clusters present in the solution, which grow preferentially in the regions of highly intensified plasmon fields at the $\mathrm{Au}$ NP poles determined by the direction of polarization. As the Au NPs grow, their surface plasmon resonance shifts away from the $532 \mathrm{~nm}$ laser. This in turn leads to lower near field 
intensities and the reaction eventually stops, when the larger axis reaches approximately $100 \mathrm{~nm}$ (further details in the SI, Figure S3).

In addition to the photo-induced reduction, other physical phenomena are plausible to play a role. As soon as a small but polarizable Au NP forms near the pole of the immobilized AuNP, it would be attracted to the larger NP due to optically induced dipole-dipole interaction. The high temperature due to light absorption would then stimulate coalescence. ${ }^{32}$ This temperature increase does not play a dominant role in the reaction growth. Since the temperature of the Au NPs is homogeneous over its surface, a thermally induced reaction would lead to isotropic and polarization independent growth, in contrast to our observations. Moreover, melting or reshaping of the NPs due to the light induced heating can be ruled out considering the temperatures reached. This is further proved in a control experiment where printed Au NPs are irradiated in water but in the absence of gold precursor, and no changes in shape or size are observed (see Figure S1).

In summary, we have shown that spherical Au NPs immobilized by optical printing can be grown to definite sizes, one by one, by illuminating them at their plasmon resonance in the presence of $\mathrm{HAuCl}_{4}(\mathrm{aq})$. Further, we found conditions for which the photo-induced growth is faster in the direction imposed by the polarization of light, leading to spheroidal NPs of predetermined orientation. These findings are particularly interesting in the context of nanofabrication of plasmonic arrays and devices based on colloidal NPs, because they enable the controlled and in-situ anisotropic shaping of supported NPs in particular directions ${ }^{11,31,36}$. From here on, we envision the use of optical printing for systematic investigations of plasmon-assisted chemical reactions performed on supported metallic NPs. Studies with different metal NPs and reagents, as well as further exploration of light dosage, will lead to greater fundamental 
understanding and eventually, to reliable methods for the in-situ fabrication of multi-metallic NPs and directional re-shaping of NPs in well-ordered arrays.

\section{ASSOCIATED CONTENT}

\section{Supporting Information.}

Details about the materials and methods. This material is available free of charge via the Internet at http://pubs.acs.org.

\section{AUTHOR INFORMATION}

\section{Corresponding Author}

* fernando.stefani@cibion.conicet.gov.ar

\section{Present Addresses}

$\dagger$ The Blackett Laboratory, Department of Physics, Imperial College London, London SW7 2AZ, UK

\section{Funding Sources}

This project was funded with the support of the following grants: CONICET PIO 13320130100199CO, ANCYPT PICT-2009-0110, PICT-2010-2511, PICT-2013-0792, and a Partner Group of the Max-Planck-Society.

\section{Notes}

The authors declare no competing financial interest. 


\section{ACKNOWLEDGMENT}

Authors would like to thank Martin Bordenave and Federico Barabas for experimental assistance, and María Claudia Marchi for the FE-SEM imaging. ILV would like to thank Paula Angelomé and Paulo Coronel for helpful discussions, and Joe Stormer for his assistance with the manuscript proofreading.

\section{REFERENCES}

(1) Burda, C.; Chen, X.; Narayanan, R.; El-Sayed, M. a. Chem. Rev. 2005, 105 (4), 1025-1102.

(2) Coronado, E. a; Encina, E. R.; Stefani, F. D. Nanoscale 2011, 3 (10), 4042-4059.

(3) Noguez, C. J. Phys. Chem. C 2007, 111 (10), 3806-3819.

(4) Noguez, C. Opt. Mater. (Amst). 2005, 27 (7), 1204-1211.

(5) Liz-Marzán, L. M. Langmuir 2006, 22 (1), 32-41.

(6) Long, N. N.; Vu, L. Van; Kiem, C. D.; Doanh, S. C.; Nguyet, C. T.; Hang, P. T.; Thien, N. D.; Quynh, L. M. J. Phys. Conf. Ser. 2009, 187, 012026.

(7) Xia, Y.; Halas, N. MRS Bull. 2005, 30 (5), 338-348.

(8) Enustun, B. V.; Turkevich, J. Jacs 1963, 85 (21), 3317-3328.

(9) Kettemann, F.; Birnbaum, A.; Witte, S.; Wuithschick, M.; Pinna, N.; Kraehnert, R.; Rademann, K.; Polte, J. Chem. Mater. 2016, 28 (11), 4072-4081.

(10) Wuithschick, M.; Birnbaum, A.; Witte, S.; Sztucki, M.; Vainio, U.; Pinna, N.; Rademann, K.; Emmerling, F.; Kraehnert, R.; Polte, J. J. ACS Nano 2015, 9 (7), 7052-7071.

(11) Chen, C. J.; Osgood, R. M. Phys. Rev. Lett. 1983, 50 (21), 1705-1708.

(12) Jin, R.; Cao, Y. C.; Hao, E.; Métraux, G. S.; Schatz, G. C.; Mirkin, C. a. Nature 2003, 425 
(6957), 487-490.

(13) Zhang, J.; Li, S.; Wu, J.; Schatz, G. C.; Mirkin, C. A. Angew. Chemie - Int. Ed. 2009, 48 (42), 7787-7791.

(14) Zhang, J.; Langille, M. R.; Mirkin, C. A. Nano Lett. 2011, 11 (6), 2495-2498.

(15) Maillard, M.; Huang, P.; Brus, L. Nano Lett. 2003, 3 (11), 1611-1615.

(16) Redmond, P. L.; Wu, X.; Brus, L. J. Phys. Chem. C 2007, 111 (25), 8942-8947.

(17) Linic, S.; Aslam, U.; Boerigter, C.; Morabito, M. Nat. Mater. 2015, 14 (6), 567-576.

(18) Langille, M. R.; Personick, M. L.; Mirkin, C. A. Angew. Chemie - Int. Ed. 2013, 52 (52), $13910-13940$.

(19) Baffou, G.; Quidant, R. Chem. Soc. Rev. 2014, 43 (11), 3898-3907.

(20) Linic, S.; Christopher, P.; Xin, H.; Marimuthu, A. Acc. Chem. Res. 2013, 46 (8), 18901899.

(21) Urban, A. S.; Lutich, A. a.; Stefani, F. D.; Feldmann, J. Nano Lett. 2010, 10 (12), 4794 4798.

(22) Do, J.; Fedoruk, M.; J??ckel, F.; Feldmann, J. Nano Lett. 2013, 13 (9), 4164-4168.

(23) Huergo, M. A.; Maier, C. M.; Castez, M. F.; Vericat, C.; Nedev, S.; Salvarezza, R. C.; Urban, A. S.; Feldmann, J. ACS Nano 2016, 10 (3), 3614-3621.

(24) Nedev, S.; Carretero-Palacios, S.; Kirchner, S. R.; Jäckel, F.; Feldmann, J. Appl. Phys. Lett. 2014, 105 (16), 161113.

(25) Gargiulo, J.; Cerrota, S.; Cortés, E.; Violi, I. L.; Stefani, F. D. Nano Lett. 2016, 16 (2), 1224-1229.

(26) Brus, L. Acc. Chem. Res. 2008, 41 (12), 1742-1749.

(27) Zhai, Y.; DuChene, J. S.; Wang, Y.-C.; Qiu, J.; Johnston-Peck, A. C.; You, B.; Guo, W.; 
DiCiaccio, B.; Qian, K.; Zhao, E. W.; Ooi, F.; Hu, D.; Su, D.; Stach, E. A.; Zhu, Z.; Wei, W. D. Nat. Mater. 2016, 15 (8), 889-895.

(28) Brus, L. Nat Mater 2016, 15 (8), 824-825.

(29) Klar, T.; Perner, M.; Grosse, S.; von Plessen, G.; Spirkl, W.; Feldmann, J. Phys. Rev. Lett. 1998, 80, 4249-4252.

(30) Nishijima, Y.; Ueno, K.; Kotake, Y.; Murakoshi, K.; Inoue, H.; Misawa, H. J. Phys. Chem. Lett. 2012, 3 (10), 1248-1252.

(31) Kim, N. H.; Meinhart, C. D.; Moskovits, M. J. Phys. Chem. C 2016, 120 (12), 6750-6755.

(32) Bjerneld, E. J.; Murty, K. V. G. K.; Prikulis, J.; Käll, M. Chemphyschem 2002, 3 (1), 116119.

(33) Bjerneld, E. J.; Svedberg, F.; Käll, M. Nano Lett. 2003, 3 (5), 116-119.

(34) Alloyeau, D.; Dachraoui, W.; Javed, Y.; Belkahla, H.; Wang, G.; Lecoq, H.; Ammar, S.; Ersen, O.; Wisnet, A.; Gazeau, F.; Ricolleau, C. Nano Lett. 2015, 15 (4), 2574-2581.

(35) Kreibig, U.; Vollmer, M. Optical properties of metal clusters; Springer Series in Materials Science; Springer: Berlin, Heidelberg, 1995; Vol. 25.

(36) Paul, A.; Kenens, B.; Hofkens, J.; Uji-I, H. Langmuir 2012, 28 (24), 8920-8925.

Table of Contents Graphic 


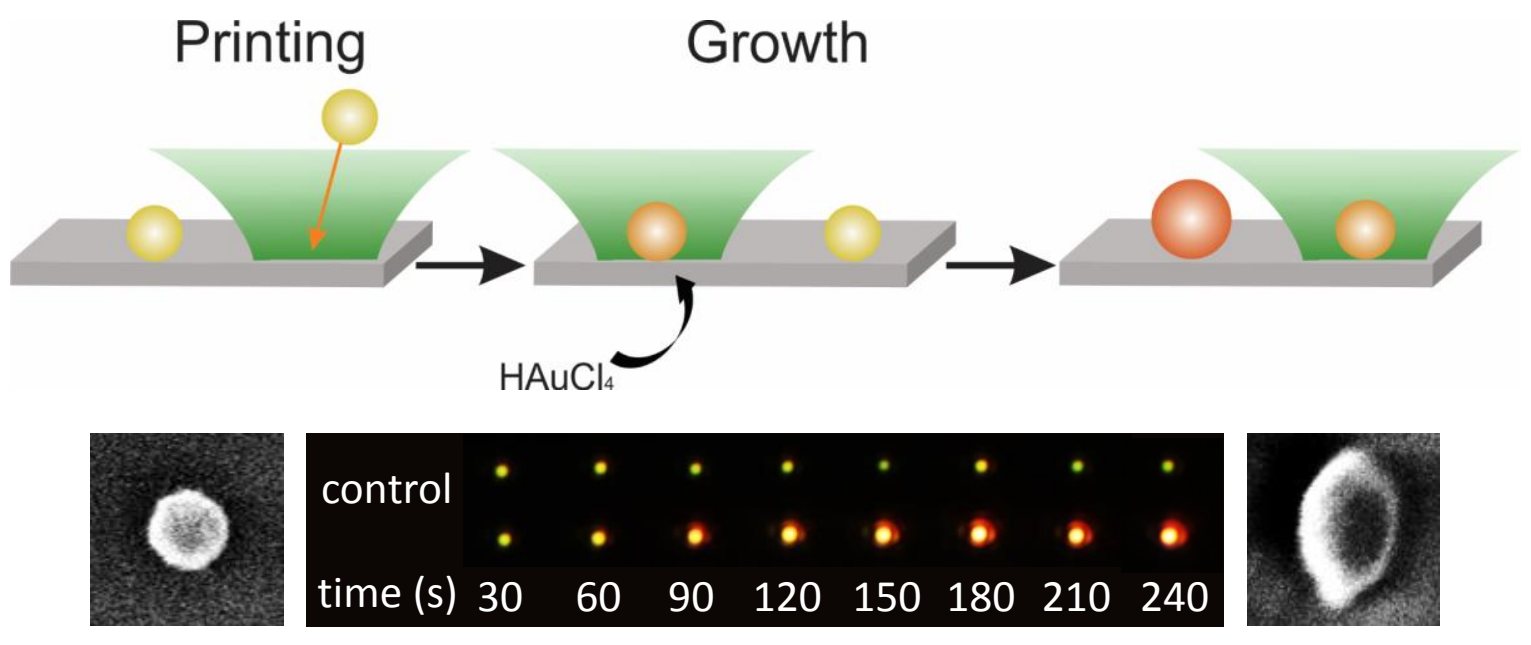

